\title{
Prevalence and Social Correlates of Sexual Intercourse among School-Going Adolescents in Thailand
}

\author{
Karl Peltzer ${ }^{1,2}$ and Supa Pengpid ${ }^{3}$ \\ ${ }^{1}$ HIVIAIDS/SIT/TB (HAST), Human Sciences Research Council, Private Bag X 41, \\ Pretoria 0001, South Africa \\ ${ }^{2}$ Department of Psychology, University of Limpopo, Turfloop, South Africa \\ ${ }^{3}$ Department of Health System Management and Policy, University of Limpopo, \\ Medunsa Campus, P.O. Box 60354, Karen Park 0118, Akasia, Pretoria, South Africa
}

Received 21 September 2011; Accepted 15 October 2011

Academic Editor: Silvia De Sanjose

\begin{abstract}
Adolescent sexuality is a relevant public health issue, as it affects risk to contract HIV and other sexually transmitted infections. The assessment of prevalence of sexual intercourse among adolescents may guide policies and programmes aimed at reducing the transmission of sexually transmitted infections among this age group. Using data from the Thailand Global School-Based Student Health Survey (GSHS) 2008, we assessed the prevalence of sexual intercourse in the last 12 months and its associated factors among adolescents $(N=2758)$. Overall the prevalence of sexual intercourse in the past 12 months was $11.0 \%$ (14.6\% males and $7.6 \%$ females). Variables positively associated with the outcome in multivariable analysis were male gender $(\mathrm{OR}=1.66 ; 95 \% \mathrm{Cl} 1.14-242)$, older age, $\geq 15$ years $(\mathrm{OR}=2.60,1.80-3.74)$, current alcohol use $(\mathrm{OR}=2.22,1.46-3.36)$, psychosocial distress $(\mathrm{OR}=2.11,1.44-3.09)$ and among females current smoking $(\mathrm{OR}=5.47,1.62-18.48)$, lifetime drug use $(\mathrm{AOR}=4.35,1.04-18.3)$ and lack of parental or guardian bonding ( $A O R=0.51,0.27-0.97$ ). Efforts to control unhealthy lifestyles (substance use) and psychosocial distress may impact on adolescents' sexual activity.
\end{abstract}

KEYWORDS: Adolescent sexuality, sexual intercourse, substance use, HIV and AIDS, protective factors, Thailand 


\section{INTRODUCTION}

Young age at first sexual intercourse is thought to increase the risk of acquiring HIV infection [1], is more likely to report sexual risk behaviours [2], and may be particularly prone to other risky behaviours later in life [3]. The adult (15-49 years) HIV prevalence in Thailand was estimated to be $1.3 \%$ in 2009 [4]. A nationwide partner relations survey conducted in 1990 found that $13 \%$ of female participants aged 1519 reported having sexual intercourse, compared with $34 \%$ of males [5]. In a local youth risk behaviour survey in Bangkok, $10 \%$ had sexual intercourse, $7.1 \%$ had never used a condom, and $2.1 \%$ resulted in pregnancy [6]. In one survey conducted in Khon Kaen, Thailand, among adolescents aged 13-15 years, $19.1 \%$ of male and $4.7 \%$ of female respondents admitted to sexual intercourse [7]. In a cross-sectional survey among 50,033 high school and vocational college students from 201 schools in 40 provinces in Thailand the rate of sexual intercourse was $30.5 \%$ for males and $5.7 \%$ for females [8]. Van Griensven et al. [9] found among 1725 students aged 15 to 21 years attending 3 vocational schools that $48 \%$ of the male students and $43 \%$ of the female students reported ever having sexual intercourse. Overall, the mean number of lifetime sexual partners was 4.6 among male participants (median: 2) and 2.8 among female participants (median: 1). Consistent use of condoms with steady partners was reported by $16 \%$ of male participants and $11 \%$ of female participants who had such partners. Among women with a history of sexual intercourse, $27 \%$ reported at least 1 pregnancy. Of these pregnancies, $83 \%$ were terminated. Among those with sexual intercourse experience, the prevalence of HIV infection was $0.5 \%$; of infection with Neisseria gonorrhoeae, $0.4 \%$; of infection with Chlamydia trachomatis, $5 \%$. Twenty-nine percent of students reported ever having used methamphetamines. Ten percent had a methamphetamine-positive urine test [9].

Various studies have identified risk factors for adolescent age at first sex. Zimmer-Gembeck and Helfand [10] integrated findings from 35 recent, longitudinal studies of the onset of heterosexual intercourse. Correlates of adolescent sexual intercourse onset, whether in early (before age 16) or middle (ages 16-18) adolescence, included living with other than two biological parents, being less monitored by parents, having more advanced physical maturity and more involvement in dating behaviour, having more permissive attitudes towards sex, alcohol use [11-15], delinquency, school problems, and (for girls) depressive symptoms. In addition, drug use (12-15), tobacco use [12, 13], male gender [13, 16], lack of parental supervision [13-15, 17], and truancy [18] were found to be associated with engaging in sexual intercourse.

There are limited reports on the prevalence of history of sexual intercourse among Thai adolescents. In order to inform public health interventions aimed to delay sexual debut and promote "safer sex," there is need for the identification of variables that are associated with adolescent intercourse. We therefore aimed to conduct this study using data from the Thailand Global School-Based Health Survey, 2008, to estimate the prevalence and correlates of having engaged in sexual intercourse in the past 12 months among in-school adolescents.

\section{METHODS}

\subsection{Participants and Procedures}

The study involved the secondary analysis of existing data from the 2008 Thailand Global School-Based Health Survey (GSHS). Details and data of the GSHS can be accessed at http://www.who.int/chp/gshs/ methodology/en/index.html. The aim of the GSHS is to collect data from students of age 13 to 15 years. The Thailand GSHS was a school-based survey of students in Grades 7, 8, 9, and 10. A two-stage cluster sample design was used to collect data to represent all students in Grades 7, 8, 9, and 10 in the country. At the first stage of sampling, schools were selected with probability proportional to their reported enrolment size. In the second stage, classes in the selected schools were randomly selected and all students in selected classes were eligible to participate irrespective of their actual ages. The school response rate was $100 \%$, the student response rate was $93 \%$, and the overall response rate was $93 \%$. Students self-completed the questionnaires to record their responses to each question on a computer scanable answer sheet. A total of 2,767 students participated in the Thailand GSHS (Ministry of Public Health). The GSHS 10 core 
questionnaire modules address the leading causes of morbidity and mortality among children and adults worldwide: tobacco, alcohol, and other drug use; dietary behaviors; hygiene; mental health; physical activity; sexual behaviors that contribute to HIV infection, other sexually transmitted infections, and unintended pregnancy; unintentional injuries and violence; protective factors and respondent demographics $[19,20]$.

\subsection{Measures}

\subsubsection{Sexual Behaviours That Contribute to HIV Infection, Other Sexually Transmitted Infections, and Unintended Pregnancy}

The sexual behaviour items were the following. "During the past 12 months, have you had sexual intercourse?" "The last time you had sexual intercourse, did you or your partner use a condom?" "How old were you when you had sexual intercourse for the first time?"

\subsubsection{Substance Use Variables}

Tobacco use: (i) smoking cigarettes (current smoking) and (ii) use of any other form of tobacco (current other tobacco use) in the past 30 days. Tobacco use was assessed with the question: "During the past 30 days, on how many days did you smoke cigarettes (use other tobacco products?" Response options included $1=0$ days to $7=$ all 30 days). Alcohol use was assessed with the question: "During the past 30 days, on how many days did you have at least one drink containing alcohol?" Response options included $1=0$ days to $7=$ all 30 days. Drug use: "During your life, how many times have you used drugs, such as methamphetamines (Yaba), ecstasy, 4 × 100, or marijuana?" (ever drugs).

\subsubsection{Psychosocial Distress Variables}

Loneliness. "During the past 12 months, how often have you felt lonely?" (Response options were from $1=$ never to $5=$ always.) (Coded $1=$ most of the time or always and $0=$ never, rarely, or sometimes.)

Anxiety or Worried. During the past 12 months, how often have you been so worried about something that you could not sleep at night? (Response options were from $1=$ never to $5=$ always.) (Coded $1=$ most of the time or always and $0=$ never, rarely or sometimes.)

Sadness. During the past 12 months, did you ever feel so sad or hopeless almost every day for 2 weeks or more in a row that you stopped doing your usual activities? (Response option $1=$ yes and $2=$ no.) $($ Coded $1=1,2=0$.

Suicide Plan. "During the past 12 months, did you make a plan about how you would attempt suicide?" (Response option was $1=$ yes and $2=$ no, coded $1=1,2=0$.)

\subsubsection{Protective Factors (School Attendance, Peer Support at School, Parental or Guardian Supervision, Connectedness, and Bonding)}

Truancy. During the past 30 days, on how many days did you miss classes or school without permission? (Response options were from $1=0$ times to $5=10$ or more times.) (Coded $1=1$ or 2 to 10 or more times, and $0=0$ times.)

Peer Support. At school was assessed with the question: "During the past 30 days, how often were most of the students in your school kind and helpful?" Parental or guardian supervision "During the past 30 days, how often did your parents or guardians check to see if your homework was done"? Parental or Guardian Connectedness: "During the past 30 days, how often did your parents or guardians understand your problems or worries?" Parental or guardian bonding: "During the past 30 days, how often did your parents or guardians really know what you were doing with your free time?" Response options to these questions were from $1=$ never to $5=$ always, $\operatorname{coded} 1=$ never or rarely, and $0=$ sometimes to always. 


\subsection{Data Analysis}

Data analysis was performed using STATA software version 10.0 (Stata Corporation, College Station, Tex, USA). This software has the advantage of directly including robust standard errors that account for the sampling design, that is, cluster sampling owing to the sampling of school classes. Psychosocial distress was assessed across the 4 mental health measures when a student's response was indicative of distress: loneliness, anxiety or worried, sadness, and suicide plan. The number of psychosocial distress indicators was calculated by determining if students had $0,1,2$, and 3 or 4 indicators [21]. Associations between sexual behaviour and sociodemographic, substance use, psychosocial distress variables, and protective factors were evaluated calculating odds ratios (ORs). Unconditional logistic regression was used for evaluation of the impact of explanatory variables for sexual intercourse in the past 12 months (binary-dependent variables). All variables statistically significant at the $P<.05$ level in bivariate analyses were included in the multivariable models. In the analysis, weighted percentages are reported. The reported sample size refers to the sample that was asked the target question. The two-sided $95 \%$ confidence intervals are reported. The $P$ values less or equal to $5 \%$ are used to indicate statistical significance. Both the reported $95 \%$ confidence intervals and the $P$ value are adjusted for the multistage stratified cluster sample design of the study.

\section{RESULTS}

Table 1 gives the sample characteristics of 2758 participants, mainly between 12 to 15 years old and $53.2 \%$ females and $46.8 \%$ males. Current smoking and current other tobacco use were reported by $8.2 \%$ and $7.2 \%$, respectively. Current alcohol use was $14.8 \%$ and lifetime illicit drug use $6.0 \%$. Sexual intercourse in the past 12 month was reported by $11 \%$ of the adolescents. Substance use and sexual behaviour variables were all higher among males than females. More than a quarter $(26.6 \%)$ of the participants had at least one psychosocial distress indicator.

Results of the bivariate and multivariable logistic regression analyses are presented in Table 2. In bivariate analyses, older age, male gender, current smoking, current other tobacco use, current alcohol use, lifetime drug use, psychosocial distress, and truancy were positively and protective factors (peer support, parental or guardian supervision, connectedness and bonding) and were inversely associated with sexual intercourse in the past 12 months. In multivariable unconditional regression analysis, older age, male gender, current alcohol use, and the number of psychosocial distress indicators were retained in this model.

\section{DISCUSSION}

The study found among in-school adolescents in Thailand using the Global School Health Survey (GSHS) of 2008 an overall prevalence of having engaged in sexual intercourse in the past 12 months of $11.0 \%$, $14.6 \%$ among male, and $7.6 \%$ among female adolescents, aged 12 to 17 years. This rate of $11 \%$ seems to be lower than in African and European countries. Data based on the Health Behaviour in School-Aged Children (HBSC) found in nationally representative samples of 15-year-olds in school classrooms in 30 mostly high-income countries that $27 \%$ of those surveyed had had sex [22]. In six African countries, using the GSHS, the prevalence of sexual intercourse in the past 12 months ranged among boys from $8.1 \%$ in Zimbabwe to $22.0 \%$ in Kenya and among girls from 3.2\% in Zimbabwe to $11.6 \%$ in Namibia [21]. In our study, males were more likely to have reported sexual intercourse and substance use (tobacco, alcohol, illicit drugs) than females. This predominance of males in potentially unhealthy lifestyles has also been found in a number of other studies, in terms of sexual intercourse, for example, in six African countries [21], Thai high school students [8], 13-15-year-old adolescents in Khon Kaen, Thailand [7], and in terms of substance use among youth in Bangkok [6], tobacco use and illicit drugs in six African countries [23].

The study further showed that adolescents who engaged in sexual intercourse in the past 12 months were also more likely to have used alcohol and among females to have smoked cigarettes and used illicit drugs. Several authors have described "clustering" of unhealthy or risk behaviours including alcohol use, 
TABLE 1: Sample characteristics among adolescents in Thailand, 2008, $N=2758$.

\begin{tabular}{|c|c|c|c|}
\hline & Total $N(\%)$ & Males $N(\%)$ & Females $N(\%)$ \\
\hline \multicolumn{4}{|l|}{ Age (years) } \\
\hline$\leq 12$ & $466(17.0)$ & $201(15.6)$ & $265(18.2)$ \\
\hline 13 & $840(29.5)$ & 407 (30.9) & $433(28.1)$ \\
\hline 14 & $870(28.7)$ & $443(30.3)$ & $427(27.2)$ \\
\hline$\geq 15$ & $582(24.9)$ & $313(23.2)$ & $269(26.5)$ \\
\hline \multicolumn{4}{|l|}{ Gender } \\
\hline Female & $1394(53.2)$ & & \\
\hline Male & $1364(46.8)$ & & \\
\hline \multicolumn{4}{|l|}{ Current smoking } \\
\hline No & $2440(91.8)$ & $1092(85.0)$ & $1348(97.8)$ \\
\hline Yes & $220(8.2)$ & $190(15.0)$ & $30(2.2)$ \\
\hline \multicolumn{4}{|l|}{ Current other tobacco use } \\
\hline No & $2555(92.8)$ & $1180(86.6)$ & 1375 (98.7) \\
\hline Yes & $201(7.2)$ & $183(13.4)$ & $18(1.3)$ \\
\hline \multicolumn{4}{|l|}{ Current alcohol use } \\
\hline No & $2126(85.2)$ & $928(78.8)$ & $1198(90.7)$ \\
\hline Yes & $368(14.8)$ & $247(21.2)$ & $121(9.3)$ \\
\hline \multicolumn{4}{|l|}{ Lifetime illicit drug use } \\
\hline No & $2579(94.0)$ & $1206(88.9)$ & $1373(98.7)$ \\
\hline Yes & $167(6.0)$ & $147(11.1)$ & $20(1.3)$ \\
\hline \multicolumn{4}{|l|}{ Sexual intercourse in the past 12 month } \\
\hline No & $2405(89.0)$ & $1123(85.4)$ & $1282(92.4)$ \\
\hline Yes & $291(11.0)$ & $189(14.6)$ & $102(7.6)$ \\
\hline \multicolumn{4}{|l|}{ Sex before 13 years } \\
\hline No & $2491(95.5)$ & $1160(92.0)$ & $1331(98.7)$ \\
\hline Yes & $116(4.5)$ & $97(8.0)$ & $19(1.3)$ \\
\hline \multicolumn{4}{|l|}{ Two or more sexual partners } \\
\hline No & $2451(94.5)$ & $1132(90.2)$ & $1319(98.4)$ \\
\hline Yes & $141(5.5)$ & $121(9.8)$ & $20(1.6)$ \\
\hline \multicolumn{4}{|l|}{ No psychosocial distress indicators } \\
\hline 0 & $1939(73.4)$ & $935(73.3)$ & $1004(73.4)$ \\
\hline 1 & $445(16.9)$ & $206(16.6)$ & $239(17.3)$ \\
\hline 2 & $192(7.1)$ & $101(8.0)$ & $91(6.4)$ \\
\hline 3 or 4 & $65(2.5)$ & $27(2.0)$ & $38(3.0)$ \\
\hline \multicolumn{4}{|l|}{ Protective factors } \\
\hline Truancy & $467(17.1)$ & $317(24.0)$ & $150(10.6)$ \\
\hline Peer support & $1123(41.7)$ & $462(34.4)$ & $661(48.5)$ \\
\hline Parental or guardian supervision & $994(35.9)$ & $469(35.2)$ & $525(36.6)$ \\
\hline Parental or guardian connectedness & $927(34.2)$ & $384(28.7)$ & $543(39.3)$ \\
\hline Parental or guardian bonding & $1214(45.4)$ & $514(38.6)$ & $700(51.8)$ \\
\hline
\end{tabular}


TABLE 2: Bivariate and multivariable logistic regression analysis of factors that are associated with sexual intercourse in the past 12 months among adolescents in Thailand, 2008.

\begin{tabular}{|c|c|c|c|c|}
\hline & Unadjusted OR (95\% CI) & $P$ value & Adjusted OR (95\% CI) & $P$ value \\
\hline \multicolumn{5}{|l|}{ Age (years) } \\
\hline$\leq 12$ & 1.00 & & 1.00 & \\
\hline 13 & $1.31(0.80-2.14)$ & 0.257 & $1.54(0.84-2.82)$ & 0.150 \\
\hline 14 & $1.53(1.03-2.28)$ & 0.036 & $1.54(0.92-2.56)$ & 0.095 \\
\hline$\geq 15$ & $2.45(1.60-3.75)$ & 0.000 & $2.60(1.80-3.74)$ & 0.000 \\
\hline \multicolumn{5}{|l|}{ Gender } \\
\hline Female & 1.00 & & 1.00 & \\
\hline Male & $2.09(1.50-2.91)$ & 0.000 & $1.66(1.14-2.42)$ & 0.012 \\
\hline \multicolumn{5}{|l|}{ Current smoking } \\
\hline No & 1.00 & & 1.00 & \\
\hline Yes & $4.07(2.67-6.19)$ & 0.000 & $1.69(0.76-3.74)$ & $0.180^{*}$ \\
\hline \multicolumn{5}{|l|}{ Current other tobacco use } \\
\hline No & 1.00 & & 1.00 & \\
\hline Yes & $4.15(2.60-6.63)$ & 0.000 & $0.82(0.36-1.87)$ & 0.610 \\
\hline \multicolumn{5}{|l|}{ Current alcohol use } \\
\hline No & 1.00 & & 1.00 & \\
\hline Yes & $4.96(3.68-6.68)$ & 0.000 & $2.22(1.46-3.36)$ & 0.001 \\
\hline \multicolumn{5}{|l|}{ Lifetime drug use } \\
\hline No & 1.00 & & 1.00 & \\
\hline Yes & $5.70(4.18-7.77)$ & 0.000 & $1.82(0.92-3.61)$ & $0.081^{*}$ \\
\hline \multicolumn{5}{|l|}{ No psychosocial distress indicators } \\
\hline 0 & 1.00 & & 1.00 & \\
\hline 1 & $2.02(1.50-2.70)$ & 0.000 & $2.11(1.44-3.09)$ & 0.001 \\
\hline 2 & $2.23(1.41-3.54)$ & 0.002 & $1.74(0.96-3.15)$ & 0.066 \\
\hline 3 or 4 & $3.89(1.95-7.76)$ & 0.001 & $2.79(1.17-6.62)$ & 0.023 \\
\hline \multicolumn{5}{|l|}{ Protective factors } \\
\hline Truancy & $2.47(1.81-3.39)$ & 0.000 & $1.09(0.58-2.05)$ & 0.783 \\
\hline Peer support & $0.77(0.62-0.97)$ & 0.030 & $1.22(0.93-1.61)$ & 0.145 \\
\hline Parental or guardian supervision & $0.52(0.36-0.76)$ & 0.002 & $0.76(0.43-1.35)$ & 0.323 \\
\hline Parental or guardian connectedness & $0.62(0.44-0.87)$ & 0.009 & $0.96(0.60-1.51)$ & 0.834 \\
\hline Parental or guardian bonding & $0.49(0.38-0.63)$ & 0.000 & $0.62(0.37-1.02)$ & $0.057^{*}$ \\
\hline
\end{tabular}

illicit drugs, and smoking $[13,14,24]$. Alcohol use has been found in various studies such as among the GSHS in six African countries to be associated with sexual intercourse and with risky sexual behaviour $[21,25]$.

The study found that psychosocial distress was associated with sexual intercourse in the past 12 months. This finding is consistent with previous research among African school-going adolescents that sexual behaviour, psychosocial distress, and substance use are interconnected [21]. We also reported that female students who perceived they had adequate parental or guardian bonding were less likely to have 
sexual intercourse than those without parental or guardian bonding. Parental protective factors have been documented for female Kenyan students [14] and from other unhealthy behaviours among adolescents in settings [17]. The connection between sexual activity and various risk factors including problem behaviour such as substance use and mental distress indicates that prevention programmes should broaden sexual health promotion and include these factors collectively in health interventions for (early) adolescents [21].

\section{LIMITATIONS OF THE STUDY}

This study had several limitations. Firstly, the GSHS only enrolls adolescents who are in school. Schoolgoing adolescents may not be representative of all adolescents in a country as the occurrence of sexual behaviour may differ between the two groups. Also, we did not assess regional and urban-rural differences in sexual behaviour. As the questionnaire was self-completed, it is possible that some study participants may have miss reported either intentionally or inadvertently on any of the questions asked. Intentional miss reporting was probably minimized by the fact that study participants completed the questionnaires anonymously. Further, the self-report of sexual behaviour should be interpreted with caution; it is possible that respondents underreported sexual behaviour, especially females. Audio-computer-assisted self-interviewing could have resulted in higher rates of reporting [9]. The questionnaire used in this study measured different concepts such as mental distress variables with single items, which are quite limited in their use as quantitative indices. A number of risk factors associated with sexual intercourse found in other studies such as school status, physical maturity, involvement in dating behaviour, attitudes towards sex, delinquency, school problems, parent factors, and characteristics of the sexual partner [2, 10, 26, 27] were not assessed and should be assessed in future studies. Furthermore, this study was based on data collected in a cross-sectional survey. We cannot, therefore, ascribe causality to any of the associated factors in the study. Prospective studies are required to follow up sexual behaviour and associated factors.

\section{CONCLUSION}

We have estimated the prevalence of sexual intercourse within the last 12 months amongst in-school adolescents in Thailand. We suggest that efforts to control adolescent alcohol consumption and psychosocial distress and among girls smoking and drug use may have an impact on adolescent sexuality.

\section{ACKNOWLEDGMENTS}

The authors are grateful to the World Health Organisation (Geneva) for making the data available to them for analysis. They also thank the Ministries of Education and Health and the study participants for making the Thailand Global School Health Survey 2008 possible, and the country survey coordinator, Dr. Pensri Kramomtong, Chief Department of Health, Ministry of Public Health. The government of Thailand and the World Health Organization did not influence the analysis, nor did they have influence on decision to publish these findings.

\section{REFERENCES}

[1] M. Ghebremichael, U. Larsen, and E. Paintsil, "Association of age at first sex with HIV-1, HSV-2, and other sexual transmitted infections among women in Northern Tanzania," Sexually Transmitted Diseases, vol. 36, no. 9 , pp. 570-576, 2009.

[2] A. Pettifor, K. O’Brien, C. MacPhail, W. C. Miller, and H. Rees, "Early coital debut and associated HIV risk factors among young women and men in South Africa," International Family Planning Perspectives, vol. 35, no. 2, pp. 82-90, 2009.

[3] E. Slaymaker, "A critique of international indicators of sexual risk behaviour," Sexually Transmitted Infections, vol. 80, no. 2, pp. ii13-ii21, 2004. 
[4] UNAIDS, UNAIDS Report on the Global AIDS Epidemic, 2010, UNAIDS, Geneva, Switzerland, 2010.

[5] W. Sittitrai, Thai Sexual Behavior and Risk of HIV Infection: A Report of the 1990 Survey of Partner Relations and Risk of HIV Infection in Thailand, Thai Red Cross Society, Bangkok, Thailand, 1999.

[6] S. Ruangkanchanasetr, A. Plitponkarnpim, P. Hetrakul, and R. Kongsakon, "Youth risk behavior survey: Bangkok, Thailand," Journal of Adolescent Health, vol. 36, no. 3, pp. 227-235, 2005.

[7] M. Kanato and K. Saranrittichai, "Early experience of sexual intercourse-a risk factor for cervical cancer requiring specific intervention for teenagers," Asian Pacific Journal of Cancer Prevention, vol. 7, no. 1, pp. 151-153, 2006.

[8] S. Assanangkornchai, A. Mukthong, and T. Intanont, "Prevalence and patterns of alcohol consumption and healthrisk behaviors among high school students in Thailand," Alcoholism: Clinical and Experimental Research, vol. 33, no. 12, pp. 2037-2046, 2009.

[9] F. van Griensven, S. Supawitkul, P. H. Kilmarx et al., "Rapid assessment of sexual behavior, drug use, human immunodeficiency virus, and sexually transmitted diseases in northern thai youth using audio-computer-assisted self-interviewing and noninvasive specimen collection," Pediatrics, vol. 108, no. 1, p. E13, 2001.

[10] M. J. Zimmer-Gembeck and M. Helfand, "Ten years of longitudinal research on U.S. adolescent sexual behavior: developmental correlates of sexual intercourse, and the importance of age, gender and ethnic background," Developmental Review, vol. 28, no. 2, pp. 153-224, 2008.

[11] W. Chaveepojnkamjorn and N. Pichainarong, "Current drinking and health-risk behaviors among male high school students in central Thailand," BMC Public Health, vol. 11, article 233, 2011.

[12] A. Liu, P. Kilmarx, R. A. Jenkins et al., "Sexual initiation, substance use, and sexual behavior and knowledge among vocational students in Northern Thailand," International Family Planning Perspectives, vol. 32, no. 3, pp. 126-135, 2006.

[13] K. C. Chinsembu, S. Siziya, A. S. Muula, and E. Rudatsikira, "Prevalence and social correlates of sexual intercourse among schoolgoing adolescents in Namibia," SAHARA J, vol. 5, no. 3, pp. 129-135, 2008.

[14] E. Rudatsikira, A. E. Ogwell, S. Siziya, and A. S. Muula, "Prevalence of sexual intercourse among school-going adolescents in Coast Province, Kenya," Tanzania health research bulletin, vol. 9, no. 3, pp. 159-163, 2007.

[15] S. Siziya, A. S. Muula, L. N. Kazembe, and E. Rudatsikira, "Harmful lifestyles' clustering among sexually active in-school adolescents in Zambia," BMC Pediatrics, vol. 8, article 6, 2008.

[16] S. Aras, S. Semin, T. Gunay, E. Orcin, and S. Ozan, "Sexual attitudes and risk-taking behaviors of high school students in Turkey: research article," Journal of School Health, vol. 77, no. 7, pp. 359-366, 2007.

[17] L. Harris, R. F. Oman, S. K. Vesely et al., "Associations between youth assets and sexual activity: does adult supervision play a role?" Child: Care, Health and Development, vol. 33, no. 4, pp. 448-454, 2007.

[18] D. Hallfors, J. L. Vevea, B. Iritani, H. Cho, S. Khatapoush, and L. Saxe, “Truancy, grade point average, and sexual activity: a meta-analysis of risk indicators for youth substance use," Journal of School Health, vol. 72, no. 5, pp. 205-211, 2002.

[19] Centers for Disease Control (CDC), "The Global School and Health Survey background," 2009, http://www.cdc .gov/gshs/background/index.2009.

[20] Ministry of Public Health Thailand, The Global School-Based Student Health Survey (GSHS) in Thailand, Ministry of Public Health Thailand, Bangkok, Thailand, 2008.

[21] R. M. Page and C. P. Hall, "Psychosocial distress and alcohol use as factors in adolescent sexual behavior among sub-saharan african adolescents," Journal of School Health, vol. 79, no. 8, pp. 369-379, 2009.

[22] S. Nic Gabhainn, A. Baban, W. Boyce, E. Godeau, and Y. Wagener, "How well protected are sexually active 15-year olds? Cross-national patterns in condom and contraceptive pill use 2002-2006," International Journal of Public Health, vol. 54, no. 2, pp. S209-S215, 2009.

[23] K. Peltzer, "Prevalence and correlates of substance use among school children in six African countries," International Journal of Psychology, vol. 44, no. 5, pp. 378-386, 2009.

[24] L. A. Palen, E. A. Smith, A. J. Flisher, L. L. Caldwell, and E. Mpofu, "Substance use and sexual risk behavior among South African eighth grade students," Journal of Adolescent Health, vol. 39, no. 5, pp. 761-763, 2006.

[25] K. C. Chinsembu, C. D. Kasanda, and C. N. Shimwooshili-Shaimemanya, "Prevalence, distribution and behavioural determinants of coital activity among High School students in Namibia: a cross-sectional study," Journal of Public Health and Epidemiology, vol. 3, no. 7, pp. 308-316, 2011. 
[26] O. J. Ekundayo, J. Dodson-Stallworth, M. Roofe et al., "The determinants of sexual intercourse before age 16 years among rural Jamaican adolescents," TheScientificWorldJOURNAL, vol. 7, pp. 493-503, 2007.

[27] R. W. Blum, "Youth in sub-Saharan Africa," Journal of Adolescent Health, vol. 41, no. 3, pp. 230-238, 2007.

\section{This article should be cited as follows:}

Karl Peltzer and Supa Pengpid, "Prevalence and Social Correlates of Sexual Intercourse among SchoolGoing Adolescents in Thailand," TheScientificWorldJOURNAL, vol. 11, pp. 1812-1820, 2011. 


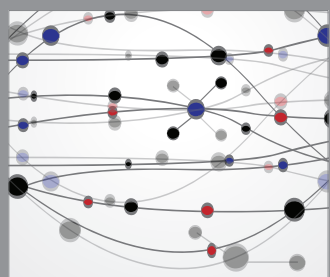

The Scientific World Journal
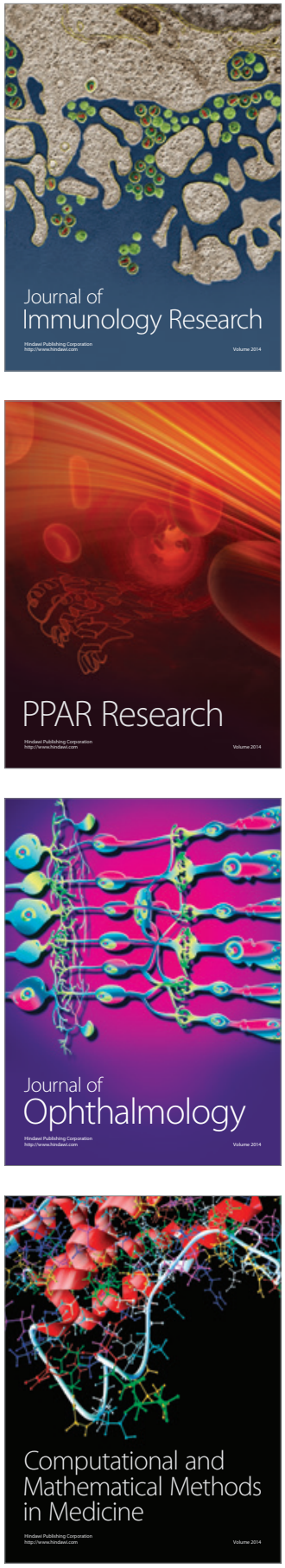

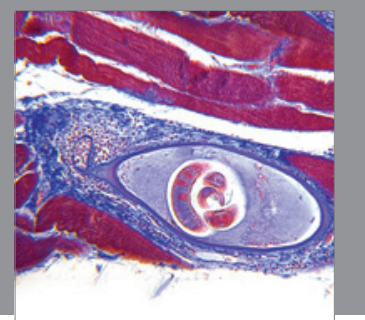

Gastroenterology

Research and Practice
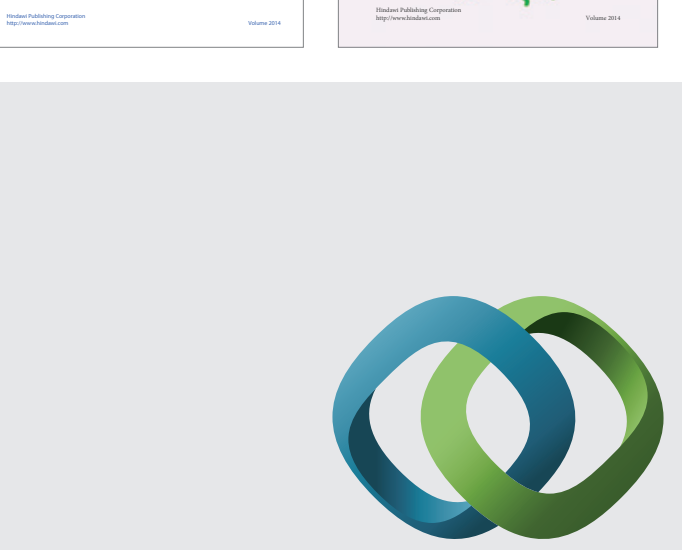

\section{Hindawi}

Submit your manuscripts at

http://www.hindawi.com
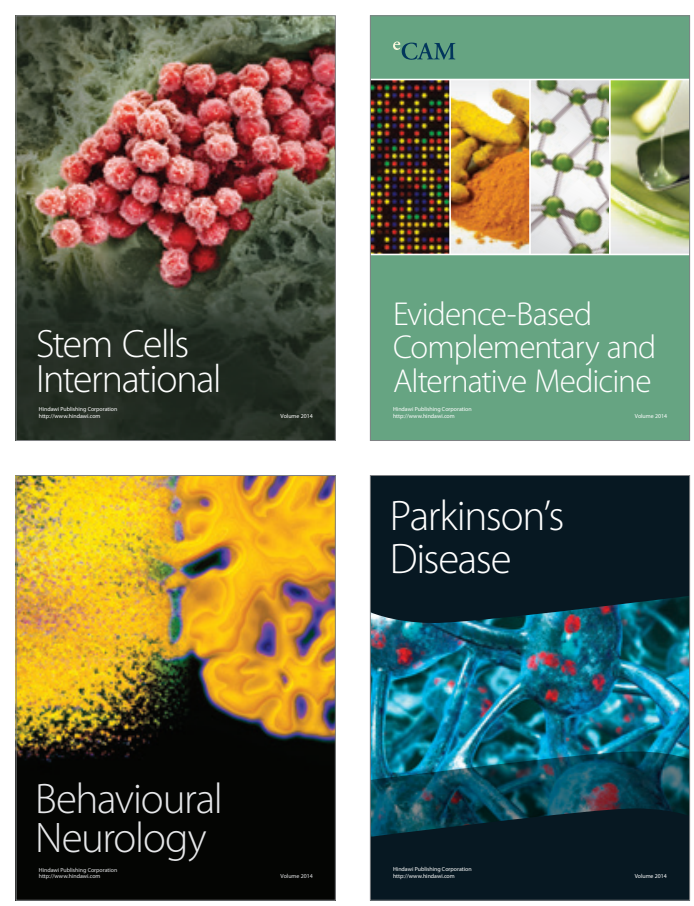

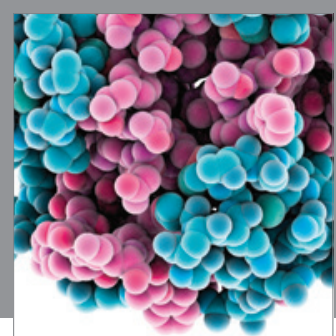

Journal of
Diabetes Research

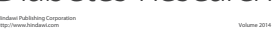

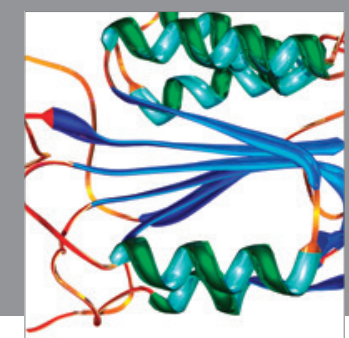

Disease Markers
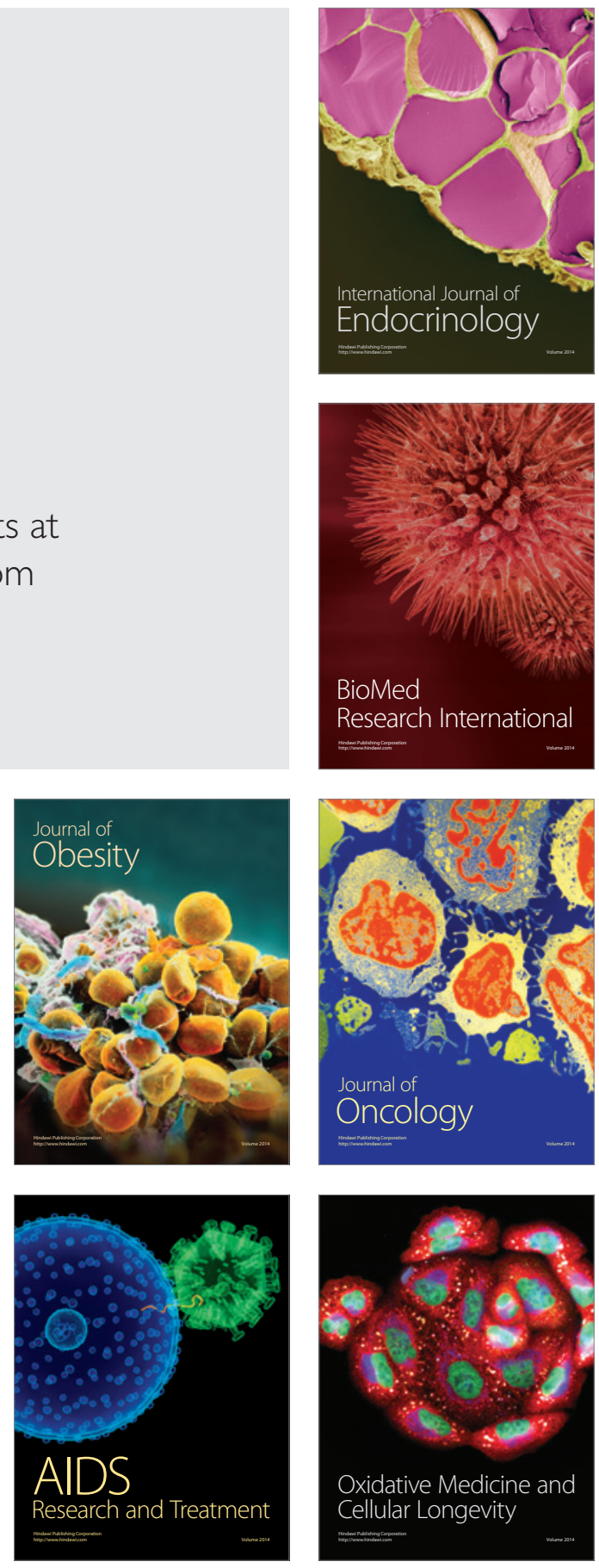